\title{
Paraneoplastic Syndromes in Children with Hodgkin Lymphoma
}

\author{
Laura Betcherman ${ }^{1}$ and Angela Punnett ${ }^{1,2}$ \\ 1. University of Toronto, Ontario, Canada; 2. Division of Hematology/Oncology, Hospital for Sick Children, Toronto, Ontario, Canada
}

DOI: https://doi.org/10.17925/OHR.2017.13.01.41

\section{Keywords}

Paraneoplastic syndrome, Hodgkin lymphoma, paraneoplastic neurological syndrome, pediatrics, malignancy, vanishing bile duct, paraneoplastic and endocrine, paraneoplastic and kidney, paraneoplastic and hematologic

Disclosure: Laura Betcherman and Angela Punnett have nothing to declare in relation to this article. This study involves a review of the literature and did not involve any studies with human or animal subjects performed by any of the authors. No funding was received in the publication of this article.

Open Access: This article is published under the Creative Commons Attribution Noncommercial License, which permits any non-commercial use, distribution, adaptation, and reproduction provided the original author(s) and source are given appropriate credit.

Received: February 22, 2017

Accepted: March 21, 2017

Citation: Oncology \& Hematology Review, 2017;13(1):41-4

Corresponding Author: Angela Punnett, Hospital for Sick Children, 555 University Ave, Toronto, ON, M5G 1X8, Canada.

E: angela.punnett@sickkids.ca
The term paraneoplastic syndrome (PNS) was first described in the 1940s as the phenomenon that certain cancers manifest as symptoms not directly related to the tumor itself.' Since then, there have been a multitude of reports on PNS, which is observed in approximately 8-10\% of all malignant tumors. ${ }^{1-3}$ PNS can affect any organ or tissue. It is generally due to ectopic hormone or cytokine production, or may be related to autoimmune factors. In some cases, the syndromes may precede the cancer diagnosis itself. ${ }^{1}$

Lymphoma is the third most common malignancy in children following leukemias and malignancies of the central nervous system. Hodgkin lymphoma ( $\mathrm{HL}$ ) occurs at a slightly higher incidence than non-Hodgkin lymphoma (NHL) and is the most common malignancy among children 15-19 years of age. ${ }^{4}$ Almost all cases arise from germinal center B cells and are associated with an inflammatory response and local disturbance of the normal function and regulation of the immune system. In this context, certain autoimmune phenomena including PNS have been described in both adults and children with HL. PNS in patients with $\mathrm{HL}$ can affect multiple organs including the neurologic, hematologic, endocrine, renal, and hepatic systems. ${ }^{5-7}$ In addition to the above, hepatic, dermatologic, and rheumatologic manifestations have also been described in adults. ${ }^{3,6,8-11}$ PNS in children with HL is relatively rare, with few published case reports and case series in this population. This article will review the state of knowledge of PNS in pediatric $\mathrm{HL}$.

\section{Neurologic syndromes}

Paraneoplastic neurologic syndromes refer to signs and symptoms resulting from target organ damage to any part of the nervous system that is not directly related to the malignant tumor or its metastases. ${ }^{12}$ They may affect the central and peripheral nervous systems, the neuromuscular junction, and the muscle itself, and may be associated with significant neurologic morbidity and mortality. Detailed diagnostic criteria for these syndromes were defined by consensus of an international panel in 2004 taking into consideration the particular syndrome, timing, and clinical outcome in relation to cancer diagnosis and therapy, and presence of onconeural antibodies..$^{13}$ The latter are supportive but not required to make a diagnosis of a paraneoplastic neurologic syndrome.

The most commonly described neurologic syndromes in children with any type of malignancy include opsoclonus-myoclonus syndrome, limbic encephalitis (LE), paraneoplastic cerebellar degeneration (PCD), and anti-N-methyl-D-aspartate (anti-NMDA) receptor encephalitis. ${ }^{12-14}$ Although opsoclonusmyoclonus syndrome is usually associated with neuroblastoma, the other three have been described in pediatric $\mathrm{HL}$ and may be associated with onconeural antibodies-anti-metabotropic glutamate receptor 5 (anti-mGluR5) in LE, the anti-Purkinje cell cytoplasmic antibody-Tr (anti-Tr) in cerebellar 
degeneration, and anti-NMDA antibody in anti-NMDA encephalitis. ${ }^{6,12}$ Although the pathophysiology remains unclear, it has been speculated that the expression of these onconeural proteins by the lymphoma triggers an immune response that is misdirected to the patient's nervous system, resulting in the associated deficits. ${ }^{13}$

\section{Limbic encephalitis}

The association of LE with $\mathrm{HL}$ is known as Ophelia syndrome. ${ }^{15}$ Typical features include personality changes, irritability, cognitive dysfunction, frank psychosis, generalized or complex partial seizures, and memory loss. There are no clear-cut diagnostic criteria for LE in children; in adults, the definition requires:

- subacute onset (up to 12 weeks) of seizures, short-term memory loss, confusion, and psychiatric symptoms;

- neuropathologic or neuroradiologic evidence of involvement of the medial temporal lobes and amygdala;

- exclusion of other possible etiologies of the limbic system; and

- demonstration of a cancer within 5 years of the diagnosis of the neurologic disorder or demonstration of an onconeural antibody. . $^{6} 15$

LE is an autoimmune process and the anti-mGLuR5 antibody is the associated onconeural antibody found in $60 \%$ of adult patients. ${ }^{6}$ The incidence in children is unknown. Because the autoimmune process in paraneoplastic LE is more commonly associated with neuronal dysfunction than neuronal death, LE in patients with HL typically has a better prognosis. Patients often have a full neurologic recovery with successful treatment of the tumor. ${ }^{12}$ Other attempted therapies have included early immunotherapy with intravenous immunoglobulin, steroids, and plasma exchange, and in more refractory cases, cyclophosphamide, and monoclonal anti-B cell antibodies. However, it should be noted that the treatment response in these cases is poor, especially in those patients with detectable onconeural antibodies. ${ }^{7,12,15}$

\section{Paraneoplastic cerebellar degeneration}

PCD is the best characterized paraneoplastic neurologic syndrome in pediatric $\mathrm{HL}$. The hallmark of PCD is a loss of Purkinje cells in the cerebellum, leading to subacute dysfunction. It presents with dizziness and vertigo, followed by truncal and limb ataxia, dysarthria, diplopia, and downbeating nystagmus. Imaging studies demonstrate evidence of cerebellar atrophy on magnetic resonance imaging (MRI) and hypometabolism on positron emission tomography (PET) scan. Lumbar puncture may reveal a cerebrospinal fluid (CSF) pleocytosis. In approximately $80 \%$ of adult and pediatric patients with HL, PCD precedes the oncologic diagnosis. ${ }^{6}$

PCD is an immune-mediated syndrome, sometimes associated with the anti-Tr antibody. ${ }^{16}$ Like other PNS in pediatric $\mathrm{HL}$, the symptoms often resolve with standard therapy. Avramova et al. described an eight-year-old boy presenting with dysarthria and diplopia and subsequently diagnosed with $\mathrm{HL}$. He had a complete resolution of symptoms with standard $\mathrm{HL}$ treatment. ${ }^{14}$ Immunotherapy has not been shown to be particularly effective in the treatment of the neurologic outcomes associated with PCD. ${ }^{14,17}$

\section{Anti-N-methyl-D-aspartate receptor encephalitis}

Anti-NMDA receptor encephalitis is a recently described phenomenon that may occur as a PNS. Affected children exhibit neuropsychiatric symptoms such as behavioral and personality disturbances, sleep dysfunction, dystonias, autonomic instability, dyskinesias, and speech dysfunction or regression. ${ }^{12,18}$ The NMDA receptor is responsible for synaptic plasticity and an overactive NMDA receptor, specifically due to antibodies against the NR1 subunit, causes an excitotoxicity that is thought to mediate the observed neuropsychiatric symptoms. ${ }^{19}$ Anti-NMDA receptor encephalitis must be distinguished from primary and secondary psychoses.

Most commonly associated with germ cell tumors, there are two case reports of anti-NMDA receptor encephalitis associated with $\mathrm{HL}^{18,20}$ The diagnosis is supported by the presence of a CSF pleocytosis or oligoclonal bands, the presence of the anti-NMDA receptor in the CSF, electroencephalogram (EEG) findings of slowed activity, and typical contrast-enhanced MRI findings. ${ }^{19}$ In the case of PNS, successful tumor treatment in addition to steroids, Intravenous immunoglobulin (IVIG), or plasma exchange tends to resolve the neuropsychiatric symptoms. ${ }^{18}$ Second-line therapy should be considered if there is no significant improvement after 10 days. A 4-week course of anti-B cell monoclonal antibody therapy or cyclophosphamide may be attempted. ${ }^{18}$

\section{Other neurologic manifestations}

There are several other neurologic manifestations of PNS in pediatric HL. Srinivasan et al. published a case report of a child with $\mathrm{HL}$ presenting with two rare paraneoplastic neurologic syndromes: Holmes-Adie pupil and achalasia. ${ }^{16}$ In this case, they postulated that both manifestations were secondary to immune-mediated damage to the ganglion cells in the myenteric plexus, further proving that PNS can manifest in any number of tissues; in this case, the autonomic system. There was one prior published case of achalasia in a child with HL. ${ }^{21}$ Although not reported in the pediatric literature, Graus et al. have collated reports of myelopathy, neuronopathies, Guillain-Barre, and neuromuscular junction disease (NMJ) disorders from the adult $\mathrm{HL}$ literature. ${ }^{\circ}$

\section{Hematologic syndromes}

The most common paraneoplastic hematologic syndromes seen in $\mathrm{HL}$ include autoimmune hemolytic anemia (AlHA) and immune thrombocytopenia (ITP). ${ }^{7}$ There are only a few case reports of AlHA and ITP in pediatric HL. Ertem et al. published a case of autoimmune bi-cytopenia as a presentation of Hodgkin disease in a 6-year-old boy, one of the two case reports where AlHA and ITP co-occurred in HL. ${ }^{22}$ Marino et al. presented the case of a 16-year-old presenting with thrombocytopenia. ${ }^{23}$ The increased megakaryocytes on bone marrow aspirate, and negative viral serology were suggestive of ITP. After the first course of chemotherapy, the platelet count rose to within normal values. In addition, they report on four additional cases of paraneoplastic ITP with and without AlHA in HL treated with chemotherapy and autoimmune disease therapy, including immunoglobulins and steroids. Although it may be difficult to differentiate either AIHA or ITP as being an independent disorder versus a paraneoplastic phenomenon, the identification of ITP or AlHA prior to HL diagnosis and remission with cancer chemotherapy suggest a paraneoplastic mechanism. ${ }^{23}$

\section{Renal syndromes}

In adults, glomerulonephritis and amyloidosis have been reported as paraneoplastic renal syndromes in $\mathrm{HL}$. . In children, nephrotic syndrome (NS) is most commonly described in the context of $\mathrm{HL}$ although the incidence remains less than 1\%. ${ }^{11,24}$ More than half of the cases of NS occurred prior to or concurrent with the diagnosis of $\mathrm{HL} .{ }^{24}$ The presentation is identical to 
that of primary or secondary NS, and is characterized by hypoalbuminemia, diffuse edema, lipidemia, proteinuria, and hypercoagulability. Proteinuria may be the only presenting element of paraneoplastic NS in pediatric $\mathrm{HL} .{ }^{11}$ It is important to distinguish the paraneoplastic phenomenon from other mechanisms of renal damage in malignancy: direct infiltration, renal vein thrombosis, renal arterial compression, and biochemical electrolyte abnormalities. The most common subtype of $\mathrm{HL}$ associated with renal paraneoplastic disease in both adults and children is nodular sclerosis, and the most common renal abnormality is minimal change NS. ${ }^{24}$ The exact mechanism remains unknown, but it is postulated that immune dysfunction including abnormal cytokine secretion or immune complex deposition leading to alterations in the glomerular basement membrane may play a role..$^{11,24}$ With adequate treatment of the $\mathrm{HL}$, the prognosis is good, and the symptoms of NS resolve completely. Steroid treatment is typically ineffective; therefore, physicians should maintain a high index of suspicion for an underlying lymphoma in steroid-resistant NS in the pediatric population. Moreover, patients with $\mathrm{HL}$ should be evaluated for renal dysfunction.

\section{Endocrine syndromes}

Paraneoplastic endocrine syndromes are thought to occur from ectopic production of hormones by the tumor cells, and are typically identified after themalignancyhasbeendiagnosed. ${ }^{~}$ Syndromeofinappropriateantidiuretic hormone secretion (SIADH) and hypercalcemia have been described in the Iymphoma literature, though only the latter has been reported in the pediatric population. ${ }^{725}$ Mittra and Davidzon report a case of a 15-year-old girl presenting with weight loss, Iymphadenopathy, bilious emesis, and acute pancreatitis. ${ }^{26} \mathrm{~A}$ full diagnostic workup was conducted to rule out an intra-abdominal pathology. PET scan and biopsy confirmed the diagnosis of HL. The patient's hypercalcemia was thought to be the cause for her pancreatitis and a paraneoplastic origin was determined. There have been several proposed mechanisms behind paraneoplastic hypercalcemia including tumor production of parathyroid hormone-related peptide (PTHrP), cytokine production, and prostaglandin production. ${ }^{1,26}$ In lymphoma, the most common culprit is extrarenal calcitriol production that is independent of parathyroid hormone. Secondly, there seems to be an association between both $\mathrm{HL}$ and NHL and elevated vitamin D3 levels. In their case, Mittra and Davidzon suggested that calcium deposition in the pancreatic duct and trypsinogen activation secondary to the elevated calcium levels led to their patient's acute pancreatitis. ${ }^{26}$ Because of the proposed mechanism, these endocrine syndromes typically resolve with successful treatment of the tumor. Although not reported in this case, consideration could be made for the use of bisphosphonates or calcitonin in managing paraneoplastic hypercalcemia. ${ }^{27}$

\section{Hepatic syndromes}

Hepatic dysfunction occurs in approximately $3-13 \%$ of patients with HL. ${ }^{5}$ Although uncommon as a cause of hepatic dysfunction, idiopathic cholestasis (IC) and vanishing bile duct syndrome (VBDS) have been reported as paraneoplastic phenomenon that present with painless jaundice. ${ }^{27}$ Significant hepatic dysfunction requires careful consideration as to the choice of upfront chemotherapy agents.

\section{Idiopathic cholestasis}

IC exists within the spectrum of paraneoplastic hepatic dysfunction and has been described only in a few cases of HL., ${ }^{52}$ It has a similar presentation to VBDS but without evidence of ductopenia, and has been reported in a pediatric patient. She presented with progressive painless, non-obstructive jaundice, shown to be IC by biopsy. Corticosteroids were attempted as treatment; however, the patient died 8 months after the onset of jaundice. Portal infiltration of $\mathrm{HL}$ was found at autopsy but was deemed a probable late and terminal finding. The authors speculate that the pathogenesis is related to toxic effects of the lymphoma itself, or a viral etiology if associated with the onset of the malignancy. ${ }^{29}$

\section{Vanishing bile duct syndrome}

VBDS is a rare syndrome characterized by jaundice and hepatic laboratory abnormalities secondary to a loss of the normal biliary duct system. It is diagnosed based on documentation of hepatic dysfunction with pathognomonic histologic findings. It is important to rule out other causes for jaundice in a patient with $\mathrm{HL}$, and VBDS remains a diagnosis of exclusion. Other more common causes include biliary obstruction though mass effect of surrounding lymph nodes, hemolysis, chemotherapy complications, or viral causes. ${ }^{30,31}$

Over the past two decades, VBDS has been described in association with HL. The underlying cause for the ductopenia is unknown, but it is thought to be an immune-mediated pathogenesis. ${ }^{30} \mathrm{~A}$ second theory includes direct bile duct damage by paraneoplastic toxic cytokines released by the lymphoma cells. ${ }^{31}$ Without treatment, the natural progression is hepatic failure. In cases of VBDS that are not related to $\mathrm{HL}$, the bile ducts disappear irreversibly; however, as a paraneoplastic phenomenon, full resolution of symptomatology, laboratory, and histologic findings has been described with appropriate $\mathrm{HL}$ therapy in some case reports.,50,31 Two reviews of $\mathrm{HL}$ and VBDS in both adults and children reported severe complications, notably liver failure and sepsis, and high mortality rates. The addition of steroid therapy to standard chemotherapy and radiotherapy improved hepatic function and was more likely to lead to complete remission. The use of high-dose ursodeoxycholic acid (UDCA) has also been proposed..$^{30}$ The addition of autologous stem cell transplantation may be another beneficial treatment approach. ${ }^{31}$

\section{Rheumatologic syndromes}

There have been few published case reports of paraneoplastic rheumatologic syndromes in $\mathrm{HL}$. There is speculation that chronic antigenic stimulation in the context of inflammatory disease may predispose to lymphoproliferative disease, and thus rheumatologic manifestations may precede the cancer diagnosis. Alternately, the clinical presentation may be secondary to immune phenomena or tumor cytokines. Those that exist in the adult literature include: polyarthritis, dermatomyositis and polymyositis, and vasculitis such as granulomatosis with polyangiitis. ${ }^{2}$ The pathogenesis of arthritis has been associated with circulating immune complexes that cross-react with tumor antigens. This may lead to inflammation at the synovial membrane and clinical arthritis. The vasculitides may result from tissue and vessel inflammation secondary to direct toxicity of tumor cell cytokines including TNF- $\alpha^{32}$ Although most case reports exist in adults, Shay et al. present an adolescent boy with symmetrical proximal muscle weakness, periorbital swelling, and fatigue. Based on elevated serum markers and an electromyography consistent with inflammatory muscle disease, a diagnosis of dermatomyositis was made. His symptoms were managed with prednisone. Unilateral Iymphadenopathy followed which was determined to be stage IA HL. ${ }^{32}$ As evidenced in the case, treating the autoimmune symptoms with systemic corticosteroids may mask the presenting symptoms of lymphoma and further delay the diagnosis. 


\section{Dermatologic syndromes}

Paraneoplastic itch has been defined by the Special Interest Group of the International Forum on the Study of Itch (IFSI) as a systemic reaction to the presence of a tumor or hematological malignancy neither induced by the local presence of tumor cells nor by tumor therapy and that usually disappears with remission status. 27,33 The symptom precedes the diagnosis or occurs early in the clinical course of the malignancy. Patients may present with secondary skin findings as a result of scratching, including excoriations, hyper- and hypopigmented lesions, lichenification, prurigo nodules, and scars. ${ }^{34}$ Paraneoplastic itch may occur in up to $30 \%$ of adults with $\mathrm{HL}$ and has been suggested as a fourth ' $B$ ' symptom. ${ }^{35}$ The incidence in children is unknown but there are published case reports in adolescents. ${ }^{36,37}$ The pathophysiology is poorly understood but may be histamine-mediated in keeping the eosinophilia and increased IgE levels seen in $\mathrm{HL}$ and with clinical responses to histamine blockers. Alternatively, paraneoplastic itch may be mediated through cytokines other than histamine. ${ }^{34,36}$
A number of other dermatologic PNS have been reported in adult literature, most commonly new-onset eczema, erythema nodosum, and mycosis fungoides. ${ }^{38}$

\section{Conclusion}

Paraneoplastic syndromes are relatively uncommon in $\mathrm{HL}$ but may affect many organ systems. There are few reports in children to inform the epidemiology, pathophysiology, and management of these syndromes. Clinicians must maintain a high index of suspicion for an underlying malignancy with new and unexplained clinical presentations to prevent diagnostic delay. Appropriate cancer therapy appears to be the most effective treatment for many of these syndromes but may be complicated by organ dysfunction caused by the PNS. Any adjuvant therapy should be targeted to the specific type of PNS. Dedicated clinical registries and biobanking are required for further research in this area. $\square$
1. Pelosof LC, Gerber DE, Paraneoplastic syndromes: an approach to diagnosis and treatment, Mayo Clin Proc, 2010:85:838-54.

2. Sanin WB, Bolivar YR, Carvajal $J$, et al., Polyangiitis with granulomatosis as a paraneoplastic syndrome of B-cell lymphoma of the lacrimal gland, Case Rep Hematol, 2014;2014(713048)

3. Erlij D, Calderon B, Rivera A, et al., Polyarthritis and membranoproliferative glomerulonephritis as paraneoplastic manifestation of Hodgkin's lymphoma: a case report and literature review Rheumatol Clin, 2016:12.282-4.

4. Statistics SC, SEER Cancer Statistics Review, 1975-2013, 2015 Available at: http://seer.cancer.gov/csr/1975_2013 (accessed May 5 , 2017)

5. Ballonoff A, Kavanagh B, Nash R, et al., Hodgkin lymphomarelated vanishing bile duct syndrome and idiopathic cholestasis: statistical analysis of all published cases and literature review, Acta Oncol, 2008;47:962-70.

6. Graus $\mathrm{F}_{\text {, Arino }} \mathrm{H}$, Dalmau J, Paraneoplastic neurologic syndromes in Hodgkin and non-Hodgkin Iymphoma, Blood, 2014:123:3230-8.

7. Hagler KT, Lynch JW, Paraneoplastic manifestations of lymphoma Clin Lymphoma, 2004;5:29-36.

8. Abedi SH, Ghassami M, Molaei M, et al., Secondary sclerosing cholangitis and Hodgkin's lymphoma, Clin Med Insights Case Rep, 2015;8:83-7.

9. Gong J, Lim SW, Alopecia areata as a paraneoplastic syndrome of Hodgkin's Iymphoma: a case report, $\mathrm{Mol}$ Clin Oncol, 2014:2.596-98.

10. Racanelli V Prete M, Minoia $C$, et al., Rheumatic disorders as paraneoplastic syndromes, Autoimmun Rev, 2008:7:352-8.

11. Sfrijan, D, Tieranu I, Popa L, et al., Nephrotic syndrome, paraneoplastic syndrome associated to Hodgkin lymphoma Maedica, 2016;11:64-7.

12. Alavi S, Paraneoplastic neurologic syndromes in children: a review article, Iran J Child Neurol, 2013;7:6-14.

13. Graus F, Delattre JY, Antoine JC, et al., Recommended diagnostic criteria for paraneoplastic neurologic syndromes, J Neurol Neurosurg Psychiatry, 2004;75:1135-40.
14. Avramova BE, Hristova T, Yordanova M, et al., Cerebellar degeneration as a rare paraneoplastic syndrome in a child with Hodgkin Iymphoma, J Pediatr Hematol Oncol, 2016;38:470-2.

15. Juneja, M, Kaur S, Mishra D, and Jain S, Ophelia syndrome: Hodgkin Iymphoma with limbic encephalitis, Indian Pediatr 2015;52:335-6.

16. Srinivasan A, Satish G, Scott JX, et al., Two uncommon paraneoplastic neurologic syndromes in a child with Hodgkin Iymphoma, J Pediatr Hematol Oncol, 2016;38:473-5.

17. Dalmau J and Rosenfeld MR, Paraneoplastic syndromes of the CNS, Lancet Neurol, 2008;7:327-40.

18. Aulicka $S$, Horak O, Mrazova L, et al., Malignant catatonia due to anti-NMDA-receptor encephalitis in a 15-year-old girl: case report and summary of current knowledge, Neuropsychiatry, 2016;6:136-41

19. Dalmau J, Gleichman AJ, Hughes EG, et al., Anti-NMDA-receptor encephalitis: case series and analysis of the effects of antibodies, Lancet Neurol, 2008:7:1091-9.

20. Zandi MS, Irani SR, Follows $\mathrm{G}$, et al., Limbic encephalitis associated with antibodies to the NMDA receptor in Hodgkin lymphoma Neurology, 2009;73:2039-40.

21. Benjamin SB, Castell DO, Achalasia and Hodgkin's disease: a chance association?, J Clin Gastroenterol, 1981;3:175-8.

22. Ertem M, Uysal Z, Yavuz G, Gozdasoglu S, Immune thrombocytopenia and hemolytic anemia as a presenting manifestation of Hodgkin disease, Pediatr Hematol Oncol, 2000:17:181-5

23. Marino S, Di Cataldo A, Magro G, et al., A difficult diagnosis of Hodgkin lymphoma due to immune thrombocytopenia, Clin Case Rep, 2015;3:179-82.

24. Farruggia P, Trizzino A, Maringhini S, et al., Hodgkin lymphoma and nephrotic syndrome in childhood, Indian J Pediatr. 2010:77:1147-9.

25. Eliakim $R$, Vertman $E$, Shinhar $E$, Syndrome of inappropriate secretion of antidiuretic hormone in Hodgkin's disease, Am J Med Sci, 1986:291:126-7.
26. Mittra ES, Davidzon G, Case 207: Hodgkin lymphoma with paraneoplastic hypercalcemic pancreatitis, Radiology, 2014;272:296-300.

27. Barber NA, Bierman PJ, Recognizing unusual manifestations of Hodgkin lymphoma, Curr Hematol Malig Rep, 2012;7:186-92.

28. Atalay H, Boyuk B, Ates M, et al., Idiopathic intrahepatic cholestasis as an unusual presentation of Hodgkin's disease Case Rep Hematol, 2015;2015:1-3.

29. Bouroncle BA, Old JW, Vazques AG, Pathogenesis of jaundice in Hodgkin's disease, Arch Intern Med, 1962;110:872-83.

30. Scalabrini DR, Caravelli D, Schianca FC, et al, complete remission of paraneoplastic vanishing bile duct syndrome after successful treatment of Hodgkin's lymphoma: a case report and review of the literature, BMC Res Notes, 2014;7:1-6

31. Wong $\mathrm{KM}$, Chang $\mathrm{CS}$, Wu CC, Yin HL, Hodgkin's lymphoma-related vanishing bile duct syndrome: a case report and literature review, Kaohsiung I Med Sci, 2013:29:636-41.

32. Shay M, Braester A, Cohen I, Dermatomyositis as presenting symtom of Hodgkin's disease, Ann Hematol, 1991;63:116-8.

33. Weisshaar $\mathrm{E}$, Weiss $\mathrm{M}$, Mettang $\mathrm{T}$, et al., Paraneoplastic itch: an expert position statement from the Special Interest Group (SIG) of the International Forum on the Study of Itch (IFSI), Acta Derm Venereol, 2015;95:261-5.

34. Yosipovitch G, Chronic pruritis: a paraneoplastic sign, Demarol Ther $2010 \cdot 23(6) \cdot 590-6$

35. Gobbi PG, Attardo-Parrinello G, Lattanzio G, et al., Severe pruritis should be a B-symptom in Hodgkin's disease, Cancer, 1983;51:1934-6.

36. Bartus $C L$, Parker $S R$, Hodgkin lymphoma presenting as generalized pruritis in an adolescent, Cutis, 2011;87:169-72.

37. Shelnitz LS, Paller AS, Hodgkin's disease manifesting as prurigo nodularis, Pediatr Dermatol, 1990:7:136-9.

38. Rubenstein M, Duvic M, Cutaneous manifestations of Hodgkin's disease, Int I Dermatol, 2006:45:251-6. 